\title{
CK1a-targeting inhibits primary and metastatic colorectal cancer in vitro, ex vivo, in cell-line-derived and patient-derived tumor xenograft mice models
}

\author{
Fupeng Ren, Jingwen Zhu, Kesang Li, Yiquan Cheng, Xiyan Zhu
}

Department of Hematology and Oncology, Key Laboratory of Diagnosis and Treatment of Digestive System Tumors of Zhejiang Province, Hwa Mei Hospital, Ningbo Institute of Life and Health Industry, University of Chinese Academy of Sciences, Ningbo 315000, Zhejiang

Contributions: (I) Conception and design: F Ren; (II) Administrative support: J Zhu; (III) Provision of study materials or patients: K Li; (IV) Collection and assembly of data: K Li; (V) Data analysis and interpretation: Y Cheng; (VI) Manuscript writing: All authors; (VII) Final approval of manuscript: All authors.

Correspondence to: Jingwen Zhu. Department of Hematology and Oncology, Key Laboratory of Diagnosis and Treatment of Digestive System Tumors of Zhejiang Province, Hwa Mei Hospital, Ningbo Institute of Life and Health Industry, University of Chinese Academy of Sciences, No.41 Xibei Street, Ningbo 315000, China. Email: zhujingwen2006@163.com.

Background: Colorectal cancer (CRC) remains a leading cause of cancer-related deaths globally. Despite improved understanding of its initiation and progression, and advances in diagnostic or therapeutic strategies, the treatment of metastatic CRC remains a clinical challenge, necessitating identification of novel efficacious therapeutics with little/no toxicity to non-tumor colorectal cells. The present study investigated the effect of Epiblastin A, an adenosine triphosphate (ATP)-mediated competitive inhibitor of casein kinase $1 \alpha(\mathrm{CK} 1 \alpha)$ on the viability, proliferation, and oncogenicity of CRC cells.

Methods: Comparative evaluation of the effect of Epiblastin $A$ on $C K 1 \alpha$ in fetal human normal colonic mucosa (FHC) and CRC (HCT116, HT29, DLD1) cell lines, using western blot, immunohistochemical staining, real-time polymerase chain reaction (RT-PCR), and sulforhodamine B (SRB) cytotoxicity assays. Primary culture cells, patient-derived xenograft (PDX), and tumor xenograft mice CRC models were also employed. Kaplan-Meier plots were used for survival analysis of our CRC cohort.

Results: CRC cells aberrantly express CK1 $\alpha$ at mRNA and protein levels. This overexpression of CK1 $\alpha$ is strongly associated with worse 5 -year overall survival (OS) in patients with CRC. Epiblastin $A$ inhibits CK1 $\alpha$ and compared to its apparent non-effect on FHC cells regardless of concentration, it elicits significant dosedependent inhibition of the viability of HT29, HCT116, and DLD1 cells with a $48 \mathrm{~h} \mathrm{IC}_{50}$ of 6.8, 5.0, and $3.2 \mu \mathrm{M}$, respectively. The expression of CK1 $\alpha$ in CRC primary cultures and PDX samples, significantly correlated with Ki-67 expression, and both were attenuated by Epiblastin A. We also observed that the effect of $5 \mathrm{mg} / \mathrm{kg}$ Epiblastin $A$ on tumor volume, and body weight in the CRC PDX mice models, was similar to that of $5 \mathrm{mg} / \mathrm{kg}$ Cetuximab over the time-course of our in vivo study. In DLD1-derived tumor xenograft mice, Epiblastin $A$ with very mild effect on mice body weight, suppressed tumor volume and tumor weight in a CK1 $\alpha$-dependent manner $(\mathrm{P}=0.024)$.

Conclusions: Our results demonstrate the efficacy of Epiblastin $A$ in CRC and its potential as a putative small-molecule inhibitor of $C K 1 \alpha$ and Ki-67 signaling, which are relevant in the CRC initiation, progression and prognosis.

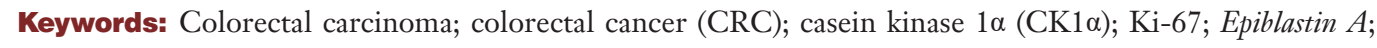
Cetuximab; chemotherapy

Submitted Sep 22, 2019. Accepted for publication Jan 16, 2020.

doi: $10.21037 /$ tcr.2020.02.13

View this article at: http://dx.doi.org/10.21037/tcr.2020.02.13 


\section{Introduction}

With an incidence of $1,849,518$ and mortality of 880,792 , colorectal cancer (CRC) accounted for $10.2 \%$ and $9.2 \%$ of all new cancer cases and cancer-related deaths, respectively, for both sexes, globally in the 2018 alone, thus, ranking as the third most diagnosed and second most fatal malignancy in the world $(1,2)$. Regardless of the significant advances made in CRC diagnosis and documented progression in the effectiveness of contemporary multimodal anti-CRC therapeutic strategies in the last 3 decades, however, their off-target toxicity against rapidly-dividing colorectal cells and acquisition of resistant phenotype continue to limit achievement of optimal therapeutic effect; thus, CRC remains associated with poor clinical outcome, with high rates of metastasis and recurrence, and relatively low overall survival (OS) in advanced-stage cases (3-7).

In the last three decades, better understanding of CRC biology and emerging kinase-related concepts reveal that the enhanced metastatic and recurrent phenotype of CRC may be associated with the enhanced activities of protein kinases, which are known to catalyze several reversible post-translational modifications, including the phosphorylation and nucleotidylation of several receptors or substrates (8-10). The human protein kinase family is sub-divided into 13 classes based on their substrates including the receptor tyrosine and serine/ threonine kinases which are critical regulators of most bioactivities including cell division, proliferation, cell fate determination, signal transduction, and cell death (8-10).

Like receptor tyrosine kinases, the transmembrane receptor serine/threonine kinases contain extracellular ligand-binding domains and cytoplasmic kinase domains. Casein kinase $1 \alpha(\mathrm{CK} 1 \alpha)$, encoded by CSNK1A1 in humans, is an isoform of the CK1 protein, exhibits broad serine/threonine kinase activity, and is a critical effector of the canonical Wnt signaling pathway (11). CK1 $\alpha$ has been shown to induce $\beta$-catenin phosphorylation at Ser45 and constitutes an active part of the $\beta$-catenin multiprotein destruction complex which subsequently facilitates the ubiquitination and proteasomal degradation of $\beta$-catenin $(11,12)$. In other studies, CK $1 \alpha$ has been shown to negatively regulate the tumor suppressor p 53 in concert with the ubiquitin ligase murine double minute clone 2 (MDM2) while enhancing E2F-1 activity in undamaged cells, thus playing a central role in $\mathrm{p} 53$ and $\mathrm{E} 2 \mathrm{~F}-1$ protein stability (13). These $\beta$-catenin-MDM2-E2F-1-p53 modulating role of CK1 $\alpha$ do suggest its probable role in the constitutive or acquired insensitivity of metastatic CRC cells to conventional CRC multimodal therapy. Thus, we hypothesized that the aberrant expression and enhanced activity of CK1 $\alpha$ is actively involved in CRC initiation, viability, proliferation, metastasis and recurrence, with the need to develop or identify therapeutic strategies that target and eliminate these CK1 $\alpha$ enriched CRC cells.

Recently, Epiblastin A, through the mediation of adenosine triphosphate (ATP), has been shown to competitively and dose-dependently inhibit CK1 at concentrations $<10 \mu \mathrm{M}$, as well as induce the dedifferentiation of mouse epiblast stem cells into embryonic stem cells several fold more efficiently than the sodium channel inhibitor, triamterene (14). While the kinase-inhibiting role of Epiblastin $A$ has attracted much scientific attention in recent years, very little is documented on its anticancer and cancer stem cells (CSCs)-targeting effect. Thus, we hypothesized based on it CK1 $\alpha$-targeting activity, that Epiblastin $A$ suppresses cancer cell division and proliferation, enhances apoptosis, inhibits tumor metastasis and recurrence, and ultimately facilitate better prognosis. This would be consistent with our increasing understanding of the critical role of protein kinase modulation and dysfunction in the progression, metastasis and recurrence of many malignancies (15) and clinically relevant considering that the single- or multiple- agent targeting of kinases is increasingly explored as an attractive therapeutic strategy for many human cancer types $(8-10,15)$.

Testing our hypotheses, in the present study, we investigated the anticancer effect of CK1 $\alpha$ in CRC in vitro (cell lines), ex vivo (clinical samples and primary culture) and in vivo [patient-derived xenograft (PDX) and tumor xenograft] models, and demonstrated that Epiblastin $A$ inhibits primary and metastatic CRC by targeting CK1 $\alpha$ and Ki-67.

\section{Methods}

\section{Patients clinicopathological characteristics}

This study was conducted on a cohort of patients with CRC at Hwa Mei Hospital, University of Chinese Academy of Sciences. The protocol for the study was reviewed and approved by the Hwa Mei Hospital, University of Chinese Academy of Sciences review board. In our CRC samples $(\mathrm{n}=78)$ from patients with CRC stage I to IV, patients aged from 29 to 72 with median age of $50.3 ; 69(88.46 \%)$ were male, while $9(11.54 \%)$ were female. The median follow-up 
time to CRC-related mortalities were recorded during the follow-up.

\section{Reagents and chemicals}

Epiblastin A ( $\geq 98 \%$ HPLC purity, CAS \#16470-02-3, Cat. \#22758) was purchased from Cayman Chemicals (Ann Arbor, MI, USA) and the anti-human EGFR monoclonal antibody, Cetuximab/Erbitux was purchased from Merck (ImClone LLC, Merck KGaA, Darmstadt, Germany). Epiblastin $A$ or Cetuximab were reconstituted following the manufacturers' instruction, stored at $-20{ }^{\circ} \mathrm{C}$, and further diluted in sterile culture medium just before use. Antibodies against CK1 $\alpha$ (sc-74582), Ki-67 (sc-23900) and glyceraldehyde 3-phosphate dehydrogenase (GAPDH) (sc47724) were purchased from Santa Cruz Biotechnology (Santa Cruz, CA, USA). Goat Alexa Fluor 488 anti-mouse IgG H \& L (ab 150113) were obtained from Abcam (Aibo Trading Co., Ltd., Shanghai, China).

\section{Cells and cell culture}

Human normal fetal colonic epithelial cell line fetal human normal colonic mucosa (FHC), colorectal carcinoma cell lines HCT116, HT29, and DLD1 were obtained from ATCC (American Type Culture Collection, Manassas, VA, USA). The cells were cultured in RPMI-1640 medium containing $5 \%$ fetal bovine serum (\#F2442, Sigma-Aldrich Inc., St. Louis, MO, USA), streptomycin $(200 \mu \mathrm{g} / \mathrm{mL})$, and penicillin (500 units/mL: Life Technologies, Grand Island, $\mathrm{NY}, \mathrm{USA}$ ) at $37^{\circ} \mathrm{C}$ in $5 \% \mathrm{CO}_{2}$ incubator.

\section{Western blotting}

After trypsinizing, harvesting and lysing CRC cells, the obtained protein lysates were then heated and used for immunoblotting. The polyvinylidene fluoride (PVDF) membranes with blots were blocked with $5 \%$ low fat milk in Tris-buffered saline (TBST) for $1 \mathrm{~h}$ and incubated with primary antibodies against CK1 $\alpha$ (1:1,000), Ki-67 (1:1,000), and GAPDH $(1: 1,000)$ overnight in $4{ }^{\circ} \mathrm{C}$ refrigerator, washed three times with TBST, incubated with horseradish peroxidase (HRP)-labeled anti-mouse secondary antibody for $1 \mathrm{~h}$ at room temperature, washed again 3 times with TBST, before protein band detection was performed using enhanced chemiluminescence (ECL) western blotting reagents and the c600 Western Blot Imaging System (Azure Biosystems Inc., Dublin, CA, USA).

\section{Sulforhodamine B (SRB) cell viability assay}

Cell viability was evaluated using SRB assay as described previously (16). Briefly, after seeding 3,500 CRC cells in complete medium in each well of 96 -well plates, the cells were incubated at $37{ }^{\circ} \mathrm{C}$ in humidified $5 \% \mathrm{CO}_{2}$ for $24 \mathrm{~h}$, followed by treatment with various concentrations of Epiblastin A, with untreated CRC cells serving as control. Our quantification was performed three times in triplicates at an optical density (OD) of $495 \mathrm{~nm}$ using SpectraMax iD3 multi-mode microplate reader (Molecular devices LLC., Shanghai Office, Shanghai, China).

\section{Quantitative real-time polymerase chain reaction ( $q R T$ - PCR)}

After total RNA isolation from tissue sections of CRC patients was performed using the RNeasy Mini Kit (QIAGEN, Hilden, Germany), reverse transcription of $1 \mu \mathrm{g}$ of total RNA into cDNA using the QuantiTect reverse transcription kit (QIAGEN, Hilden, Germany). GAPDH was used as house-keeping gene and its PCR amplification helped verify RNA integrity and cDNA synthesis fidelity based on the primer for GAPDH (forward) 5'-AGCACCAGGTGGTCTCCTCT-3' and (reverse) 5'-TGAGGTCCACCACCCTGTTG-3', and CK1 $\alpha$ (forward) 5'-AGTGGCAGTGAAGCTAGAATCT-3' and (reverse) 5'-CGCCCAATACCCATTAGGAAGTT-3'. RT-PCR was performed using the PowerUp SYBR Green Master Mix Kit (Thermo Fisher Scientific, Waltham, MA, USA) in a SimpliAmp thermal cycler (Thermo Fisher Scientific, Waltham, MA, USA) under the cycling conditions: initiation $-95^{\circ} \mathrm{C}$ for $60 \mathrm{sec}, 40$ cycles of $95^{\circ} \mathrm{C}$ for $15 \mathrm{sec}$, followed by annealing/extension for $60^{\circ} \mathrm{C}$ for $16 \mathrm{sec}$, and then reaction concluded with a melting curve analysis spanning $65-95^{\circ} \mathrm{C}$ in $0.5^{\circ} \mathrm{C}$ increments at 5 seconds per step.

\section{Immunobistochemistry assay}

Frozen paraffin-embedded samples cut into 5 - $\mu \mathrm{m}$ sections were deparaffinized by soaking in xylene thrice for 5 min each, rehydrated in $100 \%$ to $70 \%$ graded ethanol, and the slides then rinsed with distilled water for $5 \mathrm{~min}$. For hematoxylin and eosin (H\&E) staining, CRC tissues were fixed at $4{ }^{\circ} \mathrm{C}$ with $10 \%$ neutral buffered formalin overnight, transferred to $70 \%$ ethanol before being processed, paraffin-embedded, and then stained with $\mathrm{H} \& \mathrm{E}$. 
For immunochemistry, paraffin-embedded sections were incubated at $60{ }^{\circ} \mathrm{C}$ for $15 \mathrm{~min}$, deparaffinized, hydrated in $100 \%, 95 \%$ and $80 \%$ ethanol for 5 min each, rinsed in distilled water, then the slides incubated in DAKO citrate buffer ( $\mathrm{pH}$ 6.0; Agilent Technologies Inc., Santa Clara, CA, USA) were microwaved for $20 \mathrm{~min}$ to enhance antigen retrieval. Three percent hydrogen peroxide $\left(\mathrm{H}_{2} \mathrm{O}_{2}\right)$ in methanol was used to quench endogenous peroxidase activity. After the blocking process, sections were incubated at $4{ }^{\circ} \mathrm{C}$ overnight with antibodies against: CK1 $\alpha(1: 1,000)$, and Ki-67 (1:200), followed by incubation in HRPconjugated anti-mouse secondary antibodies. UltraBrite Green IHC chromogen (\#M1309, BioVision Inc., Milpitas, CA, USA) was used for detection, while hematoxylin was counterstain. Slides were read under microscope and Image analyzed using the ImageJ software.

\section{PDX and tumor xenograft assays}

Forty thousand DLD1 cells (for the tumor xenograft assays) or CRC-001/002 primary culture cells (for PDX assays) were subcutaneously injected into 5-6 weeks old NOD.CB17-Prkdc scid/NcrCrl (NOD/SCID) mice (Beijing Weitong Lihua Experimental Animal Technology Co., Ltd., China). All mice experiments were approved by the Hwa Mei Hospital, University of Chinese Academy of Sciences Animal Care and Use Committee and performed according to the institution's guidelines (HMH-2019-001). Each treatment group consisted of 10 mice. Mice were treated with $5 \mathrm{mg} / \mathrm{kg}$ Epiblastin $A$ and/or $5 \mathrm{mg} / \mathrm{kg}$ Cetuximab by intraperitoneal injection every $72 \mathrm{~h}$ after injection of tumor cells. Normal saline was used as control. The mice were sacrificed a week after the last treatment was carried out and the tumors size and weight assessed. Tumor size (in $\mathrm{mm}^{3}$ ) was calculated using the formula: $x \times y^{2} \times 0.52$, where 0.52 is a constant to calculate ellipsoid volume, $x$ is longest diameter and $y$ is the shortest diameter of tumors. Tumors from each treatment group were removed, fixed in $10 \%$ formalin and photo-images taken.

\section{Statistical analysis}

All experiments were performed three times in triplicates and data represent mean \pm standard deviation (SD). Comparison between groups was done using the student's $t$-test and one-way ANOVA with Dunnett's post hoc test. All statistical analyses were performed on the GraphPad Prism version 6.0 (GraphPad Software Inc., La Jolla, CA,
USA). $P$ value $<0.05$ was considered statistically significant.

\section{Results}

\section{CRC cells aberrantly express CK1 $\alpha$ at mRNA and protein levels}

To understand the role of CK1 $\alpha$ in CRC, we compared its expression profile in paired human colorectal tumor and normal samples, and observed that compared to its expression in normal tissues, CK1 $\alpha$ was overexpressed in the CRC samples, as demonstrated by significantly higher positive nuclear immunostaining of $\mathrm{CK} 1 \alpha$ in the tumor compared to the normal mucosal colon tissues $(\mathrm{P}<0.001)$ (Figure 1A). Using the q-PCR of oral cancer and their matched normal colon tissues from eight randomly-selected CRC patients, we also showed that the expression of CK1 $\alpha$ mRNA was upregulated in the CRC samples in comparison to the matched normal colon tissues $(\mathrm{P}<0.05)$ (Figure 1B). This was validated by the findings from our analysis of the array expression profiling data of human colon cancer cohort freely obtained from the National Center for Biotechnology Information (NCBI) Gene Expression Omnibus (GEO) profiles (http://www.ncbi.nlm.nih.gov/geoprofiles) which showed that the relative median CK1 $\alpha$ mRNA expression in human colon cancer was about 1.3-fold higher than in the normal colon samples $(\mathrm{P}<0.0001)$ (Figure $1 C$ ). In addition, we also demonstrate that compared to its expression in the human normal fetal colonic epithelial cell line FHC, the expression of CK1 $\alpha$ was significantly upregulated in CRC cell lines, HCT116, HT29, and DLD1 both at the protein (Figure 1D) and mRNA (Figure 1E) levels, using western blot analysis and q-PCR, respectively.

\section{High CK1 $\alpha$ expression is implicated in the poor clinical outcome of patients with CRC}

Because of the aberrant expression of CK1 $\alpha$ in CRC cell line and tissue samples, we hypothesized that CK1 $\alpha$ probably plays a role in the clinical outcome of patients with CRC. Thus, using the Kaplan-Meier survival curves followed by the time-stratified Mantel-Cox log-rank test, we demonstrated that patients with high CK1 $\alpha$ expression were more likely to have significantly worse 5 -year OS (1.58-fold, $\mathrm{P}=0.0379)$ or disease-free survival (DFS) (1.83-fold, $\mathrm{P}=0.0426)$, compared to their counterparts with low CK1 $\alpha$ expression levels (Figure 2A,B). Median CK1 $\alpha$ expression was used for defining high or low CK1 $\alpha$. 


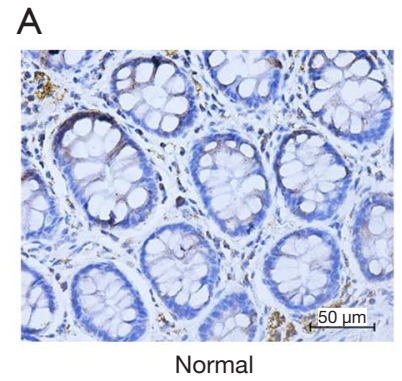

B

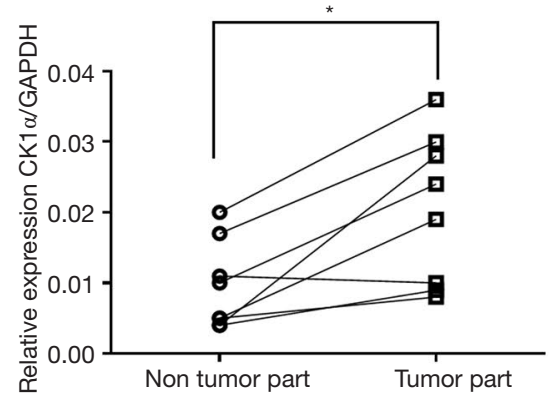

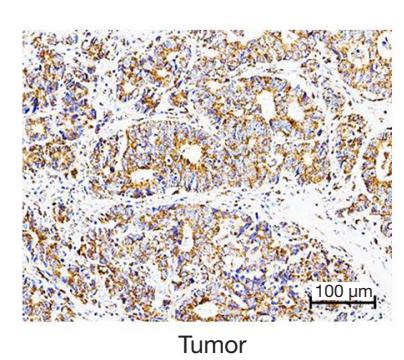

$\mathrm{D}$
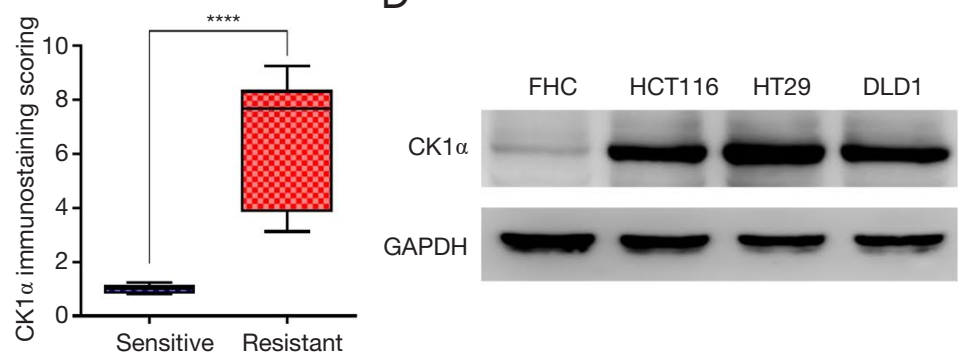

C

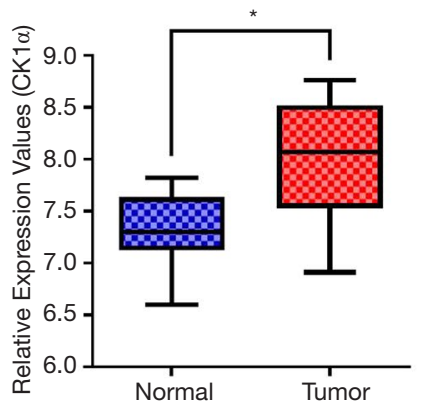

E

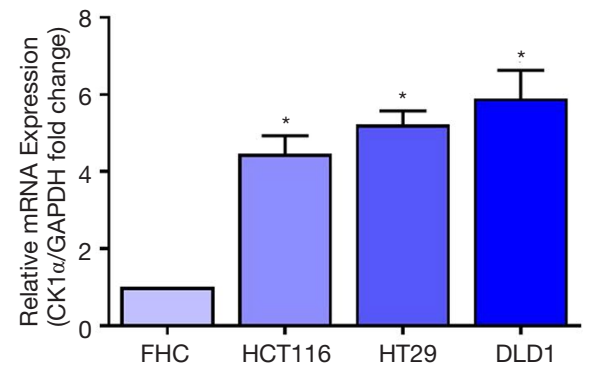

Figure 1 CRC cells aberrantly express CK1 $\alpha$ at mRNA and protein levels. (A) Immunohistochemical staining show enhanced expression of CK1 $\alpha$ protein in CRC tissue compared to the normal colon tissue; (B) the relative expression of CK1 $\alpha$ mRNA in 8 matched tumor/nontumor colonic tissues from q-PCR analysis; (C) relative expression of CK1 $\alpha$ mRNA in CRC compared to the normal colon tissue in the NCBI GEO dataset; (D) the expression levels of CK1 $\alpha$ protein in FHC, HCT116, HT29 and DLD1 cells, as shown by western blot; (E) graph of D. GAPDH served as loading control. *, $\mathrm{P}<0.05$; *** ${ }^{*} \mathrm{P}<0.0001$; data are presented as the mean $\pm \mathrm{SD}$ of experiments performed three times in triplicates. CRC, colorectal cancer; CK1 $\alpha$, casein kinase $1 \alpha$; q-PCR, quantitative polymerase chain reaction; NCBI, National Center for Biotechnology Information; GEO, Gene Expression Omnibus; FHC, fetal human normal colonic mucosa; GAPDH, glyceraldehyde 3-phosphate dehydrogenase; SD, standard deviation.

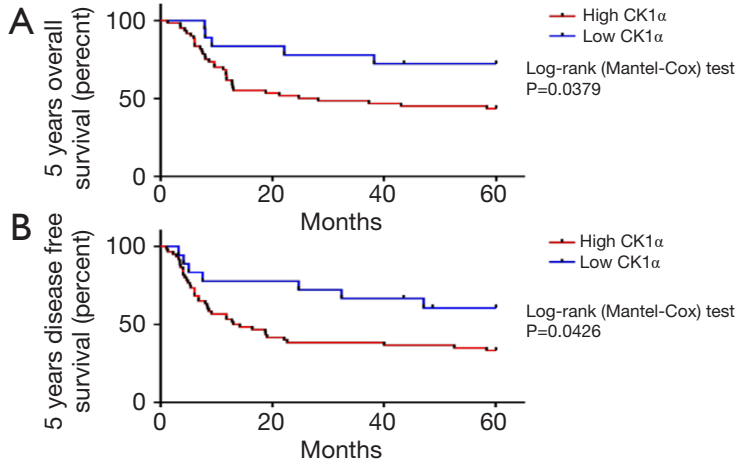

Figure 2 High CK1 $\alpha$ expression is implicated in the poor clinical outcome of patients with CRC. Kaplan-Meier curves show the effect of high CK $1 \alpha$ or low CK1 $\alpha$ on the 5-year (A) OS and (B) DFS in CRC patients. The Mantel-Cox log-rank $\mathrm{P}$ value is indicated. CRC, colorectal cancer; CK $1 \alpha$, casein kinase $1 \alpha$; OS, overall survival; DFS, disease-free survival.

\section{Pharmacological inbibition of CK1 1 using Epiblastin A significantly suppressed the viability of CRC cells without cytotoxicity to normal colonic cells, in vitro}

Having demonstrated that CK1 $\alpha$ aberrantly expressed in CRC cells and implicated in the poor clinical outcome of patients with CRC, we examined the likely anticancer effect of pharmacologically targeting CK1 $\alpha$ in CRC cells. Using FHC, HT29, HCT116 or DLD1 cells subjected to treatment with or without $0.5-25 \mu \mathrm{M}$ for $48 \mathrm{~h}$, we demonstrated that while Epiblastin $A$ had no apparent effect on the viability of FHC cells (Figure $3 A$ ), it significantly suppressed the viability of HT29 $(13.7-62.3 \%, \mathrm{P}<0.001)$ (Figure 3B), HCT116 (5.4-9.9\%, $\mathrm{P}<0.001$ ) (Figure 3C), and DLD1 (20-94.6\%, $\mathrm{P}<0.001)$ cells (Figure 3D). 

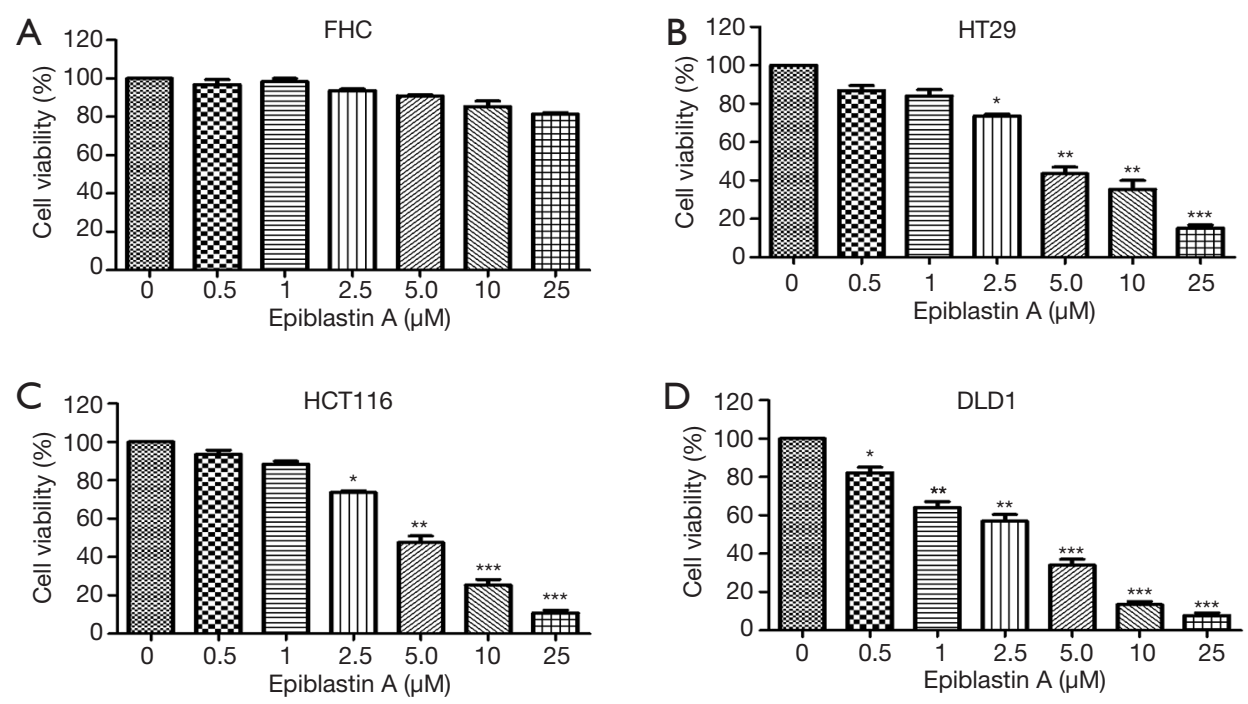

Figure 3 Pharmacological inhibition of CK1 $\alpha$ using Epiblastin A significantly suppressed the viability of CRC cells without cytotoxicity to normal colonic cells, in vitro. Histograms showing the effect of 0.5-25 $\mu$ M Epiblastin A on the viability of (A) FHC, (B) HT29, (C) HCT116, and (D) DLD1 cells. *, $\mathrm{P}<0.05$; ${ }^{* *}, \mathrm{P}<0.01$; ${ }^{* * *}, \mathrm{P}<0.001$; data are presented as the mean $\pm \mathrm{SD}$ of experiments performed three times in triplicates. CRC, colorectal cancer; CK1 $\alpha$, casein kinase $1 \alpha$; FHC, fetal human normal colonic mucosa; SD, standard deviation.

\section{Epiblastin A effectively inbibit CK1 $\alpha$ expression in CRC clinical and PDX tumor samples, ex vivo}

Evaluating the translational relevance of our findings, we performed a comparative evaluation of the expression profile of CK1 $\alpha$ in normal colon (CRC-001) versus CRC tissue (CRC-001 or CRC-002), or PDX tumor (CRC-001 or CRC-002) samples. We observed that in comparison to its no/very weak expression in the CRC-001 normal colon samples, the expression of CK1 $\alpha$ in the CRC-001 clinical or PDX tumor samples, and CRC-002 clinical or PDX tumor samples was significantly enhanced (Figure $4 A, B$ ). Subsequently, we examined the effect of treatment with $5 \mathrm{mg} / \mathrm{kg}$ Epiblastin $A$ in tissue samples derived from the PDX tumor mice. We demonstrated that treatment with $5 \mathrm{mg} / \mathrm{kg}$ Epiblastin A concurrently suppressed the expression of CK1 $\alpha$ and $\mathrm{Ki}-67$ proteins in the treated mice, compared to their untreated control counterparts (Figure 5).

\section{Epiblastin A inbibits tumor growth in CRC PDX and cell-} line-derived tumor xenograft models by suppressing CK1a expression, in vivo

In addition, for translational significance, we evaluated the therapeutic efficacy of Epiblastin A compared to the treatment of choice for patients with metastatic CRC, by using PDX tumor mice models treated with $5 \mathrm{mg} / \mathrm{kg}$ Epiblastin $A$ or same concentration of Cetuximab, After inoculation with 40,000 cells derived from CRC patient 001 or 002 , we demonstrated that without any apparently significant effect on the mice body weights, the size of tumors in mice treated with $5 \mathrm{mg} / \mathrm{kg}$ Epiblastin $A$ alone was comparable with that in the mice treated with $5 \mathrm{mg} / \mathrm{kg}$ Cetuximab alone, and significantly smaller than in the vehicle-treated mice at same time-point $(\mathrm{P}<0.05)$ (Figure 6A). This pattern was to some extent reproduced in $5 \mathrm{mg} / \mathrm{kg}$ Epiblastin A-treated CRC-002-based PDX tumor mice models (Figure 6B). Furthermore, using DLD1derived tumor xenograft models treated with or without Epiblastin A, we demonstrated that similar to its effect in the PDX models, $5 \mathrm{mg} / \mathrm{kg}$ Epiblastin $A$ markedly reduced tumor growth in the treated mice compared to the vehicletreated control group $(\mathrm{P}<0.05)$, had no apparent effect on the mice body weights, and caused a 2.18 -fold reduction in median tumor weight $(\mathrm{P}=0.04)$ over the time-course of the animal study (Figure 7A). Moreover, immunohistochemical staining of samples from the DLD1 tumor xenografts showed that the hitherto enhanced expression of CK1 $\alpha$ was profoundly suppressed in the $5 \mathrm{mg} / \mathrm{kg}$ Epiblastin $A$-treated mice (1.42-fold, $\mathrm{P}=0.02$ ) (Figure $7 B$ ). 


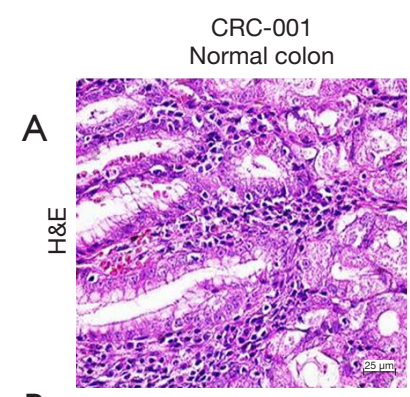

B

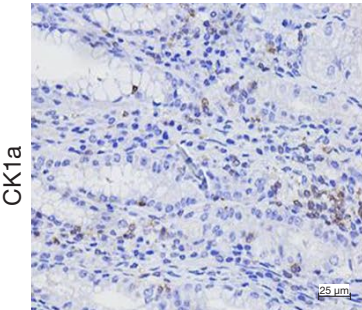

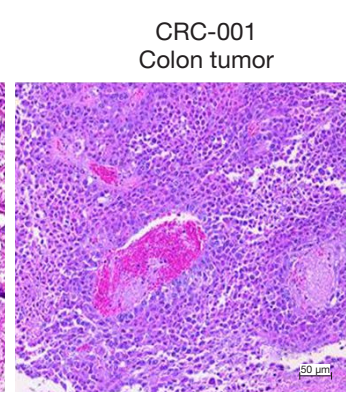

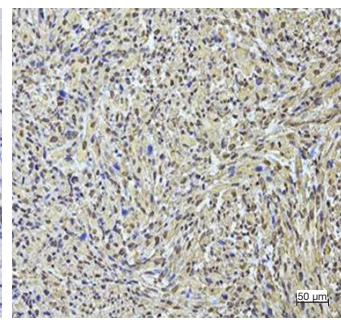

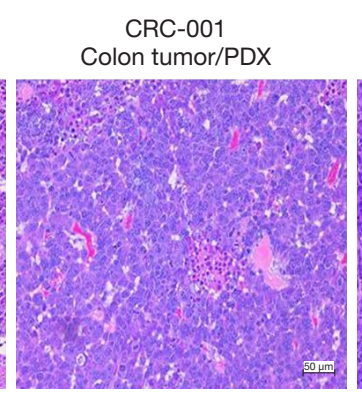

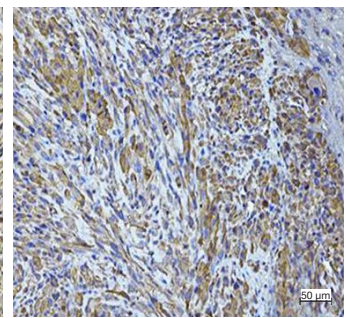

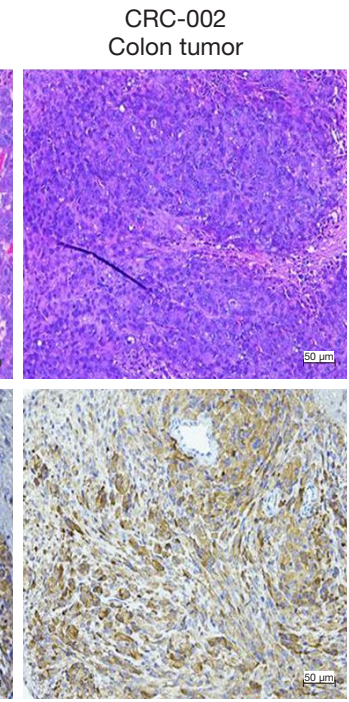

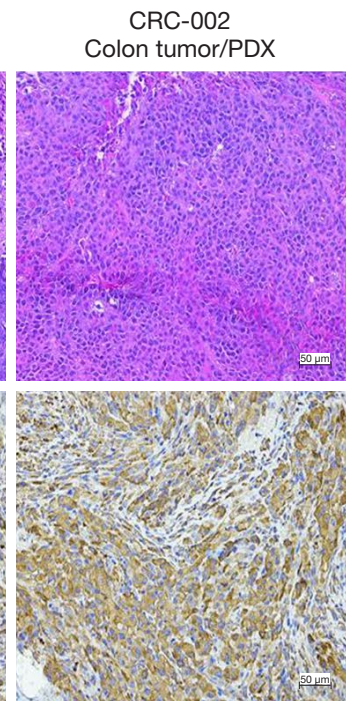

Figure 4 CK1 $\alpha$ expression is enhanced in CRC clinical and PDX tumor samples. (A) Immunohistochemical staining showing the differential expression of CK1 $\alpha$ in CRC-001 normal colon, CRC-001 colon tumor, CRC-001 colon tumor/PDX, CRC-002 colon tumor, CRC-002 colon tumor/PDX samples. H\&E was used for tissue histology; (B) quantitative representation of A. CRC, colorectal cancer; CK1 $\alpha$, casein kinase $1 \alpha$; H\&E, hematoxylin and eosin; PDX, patient-derived xenograft.
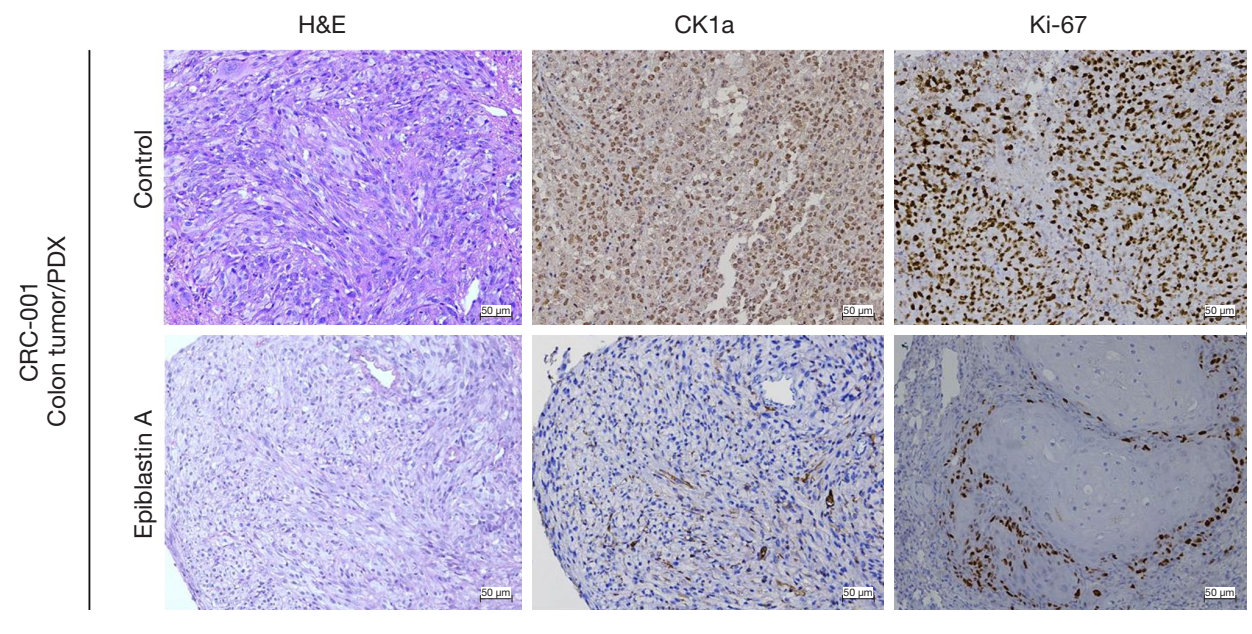

Figure 5 Epiblastin $A$ effectively inhibits CK1 $\alpha$ expression in CRC clinical and PDX tumor samples, ex vivo. (A) Immunohistochemical staining showing the effect of Epiblastin $A$ on the expression of CK1 $\alpha$ and Ki-67 in CRC-001 colon tumor/PDX samples compared to the vehicle-treated control PDX tissues. H\&E was used for tissue histology. CRC, colorectal cancer; CK1 $\alpha$, casein kinase $1 \alpha$; H\&E, hematoxylin and eosin; PDX, patient-derived xenograft.

\section{Discussion}

The overexpression of the serine/threonine kinase, CK1 $1 \alpha$, is increasingly implicated in cancer development, progression and prognosis, as evidenced by recent documentation of its role in the sustenance of oncogenic pathways including the Janus kinase/signal transducer and activator of transcription
(JAK/STAT), phosphatidylinositol-4,5-biphosphate 3-kinase (PI3K)/AKT, and nuclear factor kappa B (NF$\kappa \mathrm{B})(17)$, mediation of oncogenic autophagy in RAS-driven malignancies $(18,19)$, negative modulation of p53 and maintenance of invasive phenotype (20,21). Representing a tumorigenic weak-spot, CK1 $\alpha$ is apparently an attractive 

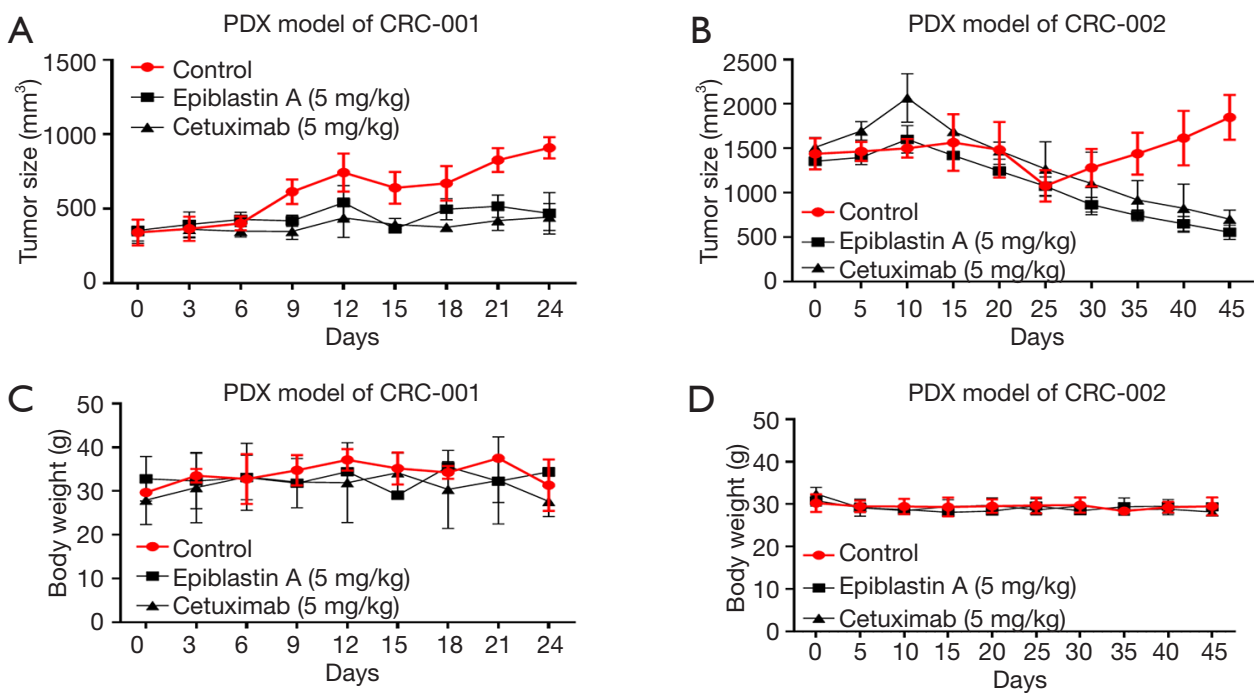

Figure 6 Epiblastin A inhibits tumor growth in CRC PDX tumor samples, in vivo. Line graphs showing the effect of $5 \mathrm{mg} / \mathrm{kg}$ Epiblastin A or Cetuximab on the size of tumors formed (upper) and mice body weights (lower) in (A,C) CRC-001 PDX mice models or (B,D) CRC-002 PDX mice models, over the indicated time-course. CRC, colorectal cancer; PDX, patient-derived xenograft.
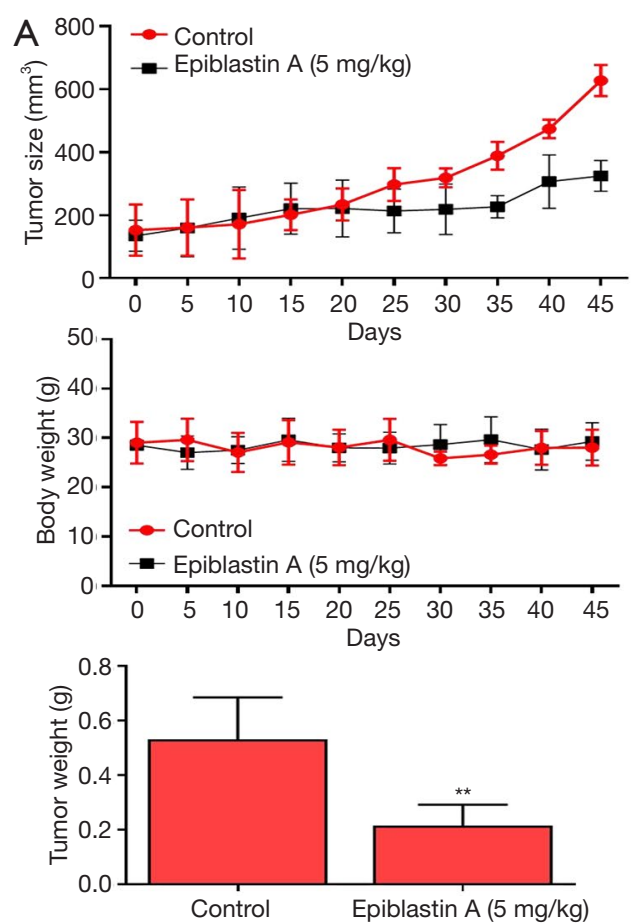

B
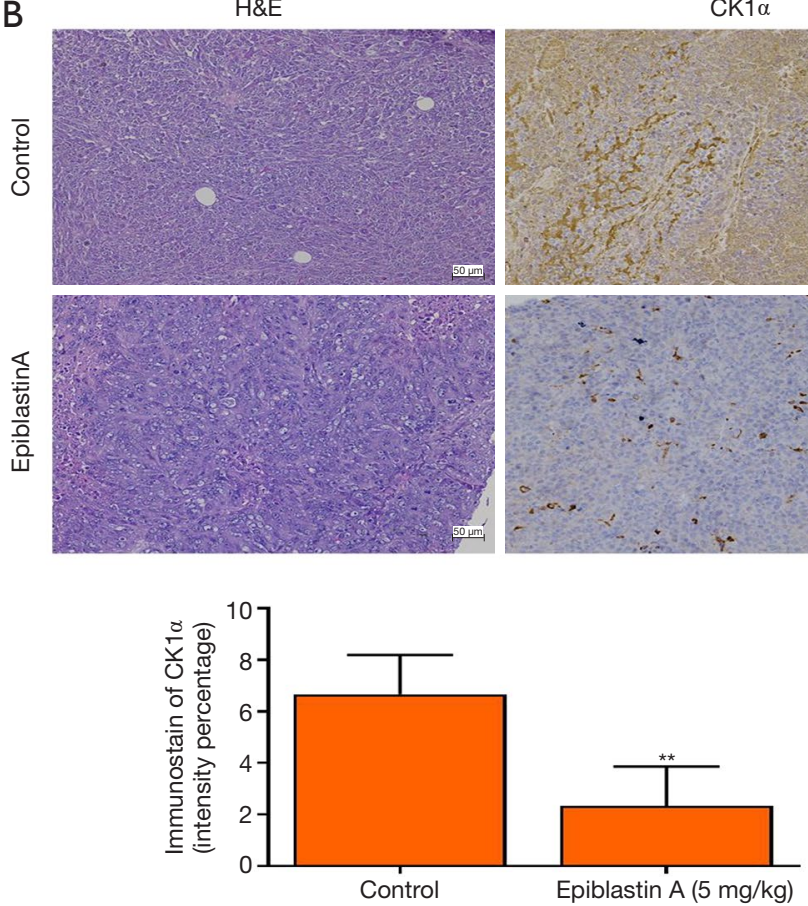

Figure 7 Epiblastin A inhibits tumor growth in DLD1-derived tumor xenograft models by suppressing CK1 $\alpha$ expression, in vivo. (A) Graphs showing the effect of $5 \mathrm{mg} / \mathrm{kg}$ Epiblastin $A$ on the size of tumors formed (upper) and mice body weights (lower) in DLD1-derived tumor xenograft mice models over the indicated time-course; (B) Immunohistochemical staining of tissue samples from the DLD1-derived tumor xenograft mice models shows the effect of Epiblastin $A$ on the expression of CK1 $\alpha$, compared to the vehicle-treated control tissues. H\&E was used for tissue histology. **, $\mathrm{P}<0.01$. CK1 $\alpha$, casein kinase $1 \alpha$; H\&E, hematoxylin and eosin. 
molecular target for effective anticancer therapy, including in CRC. Consistent with this understanding and recent demonstration of the inhibitory effect of CK1 $\alpha$ inhibition on acquired drug resistance in epidermal growth factor receptor (EGFR)-mutant non-small cell lung cancer (11), it is not out of place that the present study demonstrates that the Epiblastin $A$-induced pharmacologic targeting of CK1a inhibits primary and metastatic CRC in in vitro, ex vivo, in mice cell-line-derived, and PDX models.

Early diagnosis and adoption of effective targeted therapies based on our current knowledge of CRC biology and molecular characteristics prove to be essential to any successful treatment of patients with CRC; thus, considering the cumulative scientific evidences suggesting that the intratumoral heterogeneity and genetic constitution of CRC determine their response to targeted therapies and clinical outcome, in the present study, we demonstrated that CRC cells aberrantly express the serine/threonine kinase CK1 $\alpha$ at mRNA and protein levels (Figure 1), and that this high CK1 $\alpha$ expression is implicated in the poor clinical outcome of patients with CRC (Figure 2). This is consistent with the findings of another study which showed a positive correlation between serine threonine kinase 1 (STYK1) and disease progression, and established a functional association between the former and clinical outcome of patients with CRC as demonstrated by significantly shorter diseasespecific survival (DSS) amongst patients with high STYK1 compared to those with low STYK1 expression (22). It is worth mentioning that our findings are also corroborated by those of Richter et al. (23) showing that the overexpression of CK $1 \alpha$ correlates with poor survival in CRC based on Cox proportional hazard regression analysis of CK $1 \alpha$ and selected clinicopathological variables in a cohort of 283 CRC patients, and published while the present study was in submission.

Furthermore, based on evidence supporting the designation of Epiblastin $A$ as a potent and specific small molecule inhibitor of CK $1 \alpha$ at $\mathrm{IC}_{50} \leq 10 \mu \mathrm{M}$ (14), we demonstrated that the pharmacological inhibition of CK1 $\alpha$ using Epiblastin $A$ significantly suppressed the viability of primary CRC (HT29), transforming growth factor (TGF)- $\beta 1 / \beta 2$-rich $R A S$-mutant CRC (HCT116), and Dukes' type C p53-mutant CRC cells without cytotoxicity to normal colonic cells, in vitro (Figure 3). These finding have translational significance not only because of the proven success of recently developed small-molecule kinase inhibitors in the treatment of various types of cancer, with approximately 150 kinase-targeting drugs in clinical phase trials and about 40 kinase inhibitors already approved by the United States FDA for treatment of malignancies including for lung and breast cancers (8-10), but also because our findings show that the therapeutic efficacy of Epiblastin $A$ was not affected or limited by the presence and/or activity of $R A S$ or TGF- $\beta$, against contemporary knowledge that RAS signaling drives the switch from a tumor-suppressive to tumor-promoting role of TGF- $\beta$, leading to enhanced tumor growth and metastatic dissemination of primary tumors (24), coupled with the fact that the oncogenic $R A S$ and TGF- $\beta$ signaling induce the progression of malignancies via activation of the transcriptional program of a predominant amino-terminal-truncated isoform of the master regulator of gene expression in epithelial cells, p63 $(\Delta \mathrm{Np} 63)(25)$.

Ensuring clinical relevance, our findings were not restricted to in vitro data alone, as we also showed that Epiblastin A effectively inhibits CK1 $\alpha$ expression in CRC clinical and PDX tumor samples, ex vivo (Figures 4,5), and that Epiblastin $A$ inhibits tumor growth in CRC PDX and cell-line-derived tumor xenograft models by suppressing CK1 $\alpha$ expression, in vivo (Figure 6). While human cancerderived cell lines remain the most broadly used study models for cancer biology and preclinical cancer therapy hypothetical efficacy testing, the reliability of this model remains debatable and their clinical relevance continue to be questioned $(26,27)$. Thus, our findings replicating the in vitro Epiblastin $A$ CK $1 \alpha$-mediated anti-CRC activity ex vivo and in vivo using both cell-line-derived and PDX tumor xenograft models addresses the question of clinical relevance, and is consistent with our growing understanding that "cancer tissue represents a promising approach for the pre-clinical evaluation of conventional and immunemediated treatments and provides a platform for testing of innovative treatments" (28).

In conclusion, our findings indicate that Epiblastin $A$ inhibits primary and metastatic CRC by targeting CK $1 \alpha$ in vitro, ex vivo, and in mice cell-line-derived and PDX models. Thus, we establish the potential clinical relevance of the use of Epiblastin A as an effective anticancer treatment option in patients with CRC, regardless of nature or genetic constitution.

\section{Acknowledgments}

Funding: This work was supported by National Natural Science Foundation of China (No. 81602703), the Natural 
Science Foundation of Ningbo (No. 2017A610148). Furthermore, this work also supported by Ningbo Clinical Research Center for Digestive System Tumors (Grant No.2019A21003).

\section{Footnote}

Conflicts of Interest: All authors have completed the ICMJE uniform disclosure form (available at http://dx.doi. org/10.21037/tcr.2020.02.13). The authors have no conflicts of interest to declare.

Ethical Statement: The authors are accountable for all aspects of the work in ensuring that questions related to the accuracy or integrity of any part of the work are appropriately investigated and resolved. The study was conducted in accordance with the Declaration of Helsinki (as revised in 2013). The protocol for the study was reviewed and approved by the Hwa Mei Hospital, University of Chinese Academy of Sciences review board and informed consent was taken from all individual participants.

Open Access Statement: This is an Open Access article distributed in accordance with the Creative Commons Attribution-NonCommercial-NoDerivs 4.0 International License (CC BY-NC-ND 4.0), which permits the noncommercial replication and distribution of the article with the strict proviso that no changes or edits are made and the original work is properly cited (including links to both the formal publication through the relevant DOI and the license). See: https://creativecommons.org/licenses/by-nc-nd/4.0/.

\section{References}

1. Bray F, Ferlay J, Soerjomataram I, et al. Global cancer statistics 2018: GLOBOCAN estimates of incidence and mortality worldwide for 36 cancers in 185 countries. CA Cancer J Clin 2018;68:394-424.

2. Ferlay J, Colombet M, Soerjomataram I, et al. Estimating the global cancer incidence and mortality in 2018: GLOBOCAN sources and methods. Int J Cancer 2019;144:1941-53.

3. Duineveld LA, van Asselt KM, Bemelman WA, et al. Symptomatic and asymptomatic colon cancer recurrence: a multicenter cohort study. Ann Fam Med 2016;14:215-20.

4. Sevá-Pereira G, Cypreste RN, Oliveira Filho JJ, et al. Recurrence pattern of rectal cancer after surgical treatment. Analysis of 122 patients in a tertiary care center.
Journal of Coloproctology (Rio de Janeiro) 2018;38:18-23 .

5. Krarup PM, Nordholm-Carstensen A, Jorgensen LN, et al. Anastomotic leak increases distant recurrence and longterm mortality after curative resection for colonic cancer: a nationwide cohort study. Ann Surg 2014;259:930-8.

6. Wong CK, Law WL, Wan YF, et al. Health-related quality of life and risk of colorectal cancer recurrence and Allcause death among advanced stages of colorectal cancer 1-year after diagnosis. BMC Cancer 2014;14:337.

7. Maeda H, Kashiwabara K, Aoyama T, et al. Hazard rate of tumor recurrence over time in patients with colon cancer: implications for postoperative surveillance from three Japanese Foundation for Multidisciplinary Treatment of Cancer (JFMC) clinical trials. J Cancer 2017;8:4057-64.

8. García-Aranda $M$, Redondo $M$. Targeting receptor kinases in colorectal cancer. Cancers (Basel) 2019. doi: 10.3390/ cancers 11040433.

9. Bhullar KS, Lagarón NO, McGowan EM, et al. Kinasetargeted cancer therapies: progress, challenges and future directions. Mol Cancer 2018;17:48.

10. Gross S, Rahal R, Stransky N, et al. Targeting cancer with kinase inhibitors. J Clin Invest 2015;125:1780-9.

11. Jiang S, Zhang $M$, Sun J, et al. Casein kinase $1 \alpha$ : biological mechanisms and theranostic potential. Cell Commun Signal 2018;16:23.

12. Stamos JL, Weis WI. The $\beta$-catenin destruction complex. Cold Spring Harb Perspect Biol 2013;5:a007898.

13. Huart AS, MacLaine NJ, Meek DW, et al. CK1alpha plays a central role in mediating MDM2 control of p53 and E2F-1 protein stability. J Biol Chem 2009;284:32384-94.

14. Ursu A, Illich DJ, Takemoto Y, et al. Epiblastin A induces reprogramming of epiblast stem cells into embryonic stem cells by inhibition of casein kinase 1. Cell Chem Biol 2016;23:494-507.

15. Köstler WJ, Zielinski CC. Targeting receptor tyrosine kinases in cancer. In: Wheeler DL, Yarden Y. Receptor tyrosine kinases: structure, functions and role in human disease. New York: Springer, 2015:225-78.

16. Voigt $W$. Sulforhodamine B assay and chemosensitivity. Methods Mol Med 2005;110:39-48.

17. Manni S, Carrino M, Piazza F. Role of protein kinases CK1 $\alpha$ and CK2 in multiple myeloma: regulation of pivotal survival and stress-managing pathways. J Hematol Oncol 2017;10:157.

18. Lee SY, Kim H, Li CM, et al. Casein kinase- $1 \gamma 1$ and 3 stimulate tumor necrosis factor-induced necroptosis through RIPK3. Cell Death Dis 2019;10:923.

19. Zhang F, Virshup DM, Cheong JK. Oncogenic RAS- 
induced CK1 $\alpha$ drives nuclear FOXO proteolysis.

Oncogene 2018;37:363-76.

20. Mazzoldi EL, Pastò A, Ceppelli E, et al. Casein kinase 1 delta regulates cell proliferation, response to chemotherapy and migration in human ovarian cancer cells. Front Oncol 2019;9:1211.

21. Janovska P, Verner J, Kohoutek J, et al. Casein kinase 1 is a therapeutic target in chronic lymphocytic leukemia. Blood 2018;131:1206-18.

22. Chen S, Wang Q, Wang L, et al. REG $\gamma$ deficiency suppresses tumor progression via stabilizing CK1 $\varepsilon$ in renal cell carcinoma. Cell Death Dis 2018;9:627.

23. Richter J, Kretz AL, Lemke J, et al. CK1 $\alpha$ overexpression correlates with poor survival in colorectal cancer. BMC Cancer 2018;18:140.

24. Grusch M, Petz M, Metzner T, et al. The crosstalk of RAS with the TGF- $\beta$ family during carcinoma progression and

Cite this article as: Ren F, Zhu J, Li K, Cheng Y, Zhu X. CK1 $\alpha$-targeting inhibits primary and metastatic colorectal cancer in vitro, ex vivo, in cell-line-derived and patientderived tumor xenograft mice models. Transl Cancer Res 2020;9(3):1903-1913. doi: 10.21037/tcr.2020.02.13 its implications for targeted cancer therapy. Curr Cancer Drug Targets 2010;10:849-57.

25. Vasilaki E, Morikawa M, Koinuma D, et al. Ras and TGF- $\beta$ signaling enhance cancer progression by promoting the $\Delta \mathrm{Np} 63$ transcriptional program. Sci Signal 2016;9:ra84.

26. Gillet JP, Varma S, Gottesman MM. The clinical relevance of cancer cell lines. J Natl Cancer Inst 2013;105:452-8.

27. Gillet JP, Calcagno AM, Varma S, et al. Redefining the relevance of established cancer cell lines to the study of mechanisms of clinical anti-cancer drug resistance. Proc Natl Acad Sci U S A 2011;108:18708-13.

28. Muraro MG, Muenst S, Mele V, et al. Ex-vivo assessment of drug response on breast cancer primary tissue with preserved microenvironments. Oncoimmunology 2017;6:e1331798. 